
\title{
25 Research Suare \\ Physiological Effects of Different Artemisinin Concentrations on Highland Barley Seedlings Under Freeze-Thaw Stress
}

\section{Yue Chen}

College of New Energy and Environment, Jilin University, China

Guozhang Bao ( $\sim$ baogz@jlu.edu.cn )

College of New Energy and Environment, Jilin University, China

Jinpu Zhang

College of New Energy and Environment, Jilin University, China

Kai Lv

College of New Energy and Environment, Jilin University, China

Ruisi Fan

School of Life Science, Jilin University, China

Shasha Zheng

College of Plant Science, Jilin University, China

Xu Zhu

jilin university

Xinru Zhang

School of Science, Jilin University, China

\section{Research article}

Keywords: highland barley seedlings, artemisinin, freeze-thaw, physiological effects

Posted Date: April 13th, 2021

DOl: https://doi.org/10.21203/rs.3.rs-409646/v1

License: @ (1) This work is licensed under a Creative Commons Attribution 4.0 International License.

Read Full License 


\section{Abstract}

Background: Highland barley has good resistance to low temperature and is the most commonly used pasture and greenery in high altitude area. Beyond that, Artemisinin is an allelochemical in the common weed Artemisia annua and remains in the soil, which affects the growth and development of highland barley. Most studies on its resistance are under single stress, while plants are often affected by many factors in reality. In this paper, highland barley was used to study the effects of combined stress of simulated freeze-thaw and artemisinin with different concentrations $(10 \mathrm{mg} / \mathrm{L}, 30 \mathrm{mg} / \mathrm{L}, 50 \mathrm{mg} / \mathrm{L})$ on antioxidant enzyme activities, photosynthesis and osmotic regulation substance concentrations. The response characteristics of physiological characteristics under combined stress can study the damage mechanism of artemisinin on highland barley and provide a theoretical basis for reducing the damage caused by freeze-thaw to the growth of barley.

Results: The results showed that under freeze-thaw stress, malondialdehyde(MDA) and soluble sugar in leaves decreased significantly by $9.0-40.9 \%$ and $21.0-29.8 \%$, respectively, catalase(CAT) continued to decrease during the freeze-thaw cycle, photosynthetic rate firstly decreased and then increased, superoxide dismutase(SOD) reached the minimum value of $1470.9 \mathrm{U} / \mathrm{mgprot}$ at $-5^{\circ} \mathrm{C}$, and the soluble protein maintained a relatively stable state.As the concentration of artemisinin increased, MDA and soluble sugar firstly decreased and then increased, reaching the lowest concentration at $10 \mathrm{mg} / \mathrm{L}$. Soluble protein, CAT, SOD and photosynthetic rate first increased and then decreased.

Conclusion: All measurements indicated that under the compound stress of freeze-thaw and artemisinin, highland barley seedlings showed high cold resistance, and low concentration of artemisinin exerted a certain promotion effect on the growth of highland barley seedlings. Besides artemisinin and freeze-thaw showed an antagonistic effect on highland barley seedlings, and low concentration of artemisinin can alleviate the damage caused by freeze-thaw to highland barley seedlings.

\section{Introduction}

Highland barley (Hordeum vulgare L. Var. NudumHook. F.) is widly cultivated in the Qinghai-Tibet Plateau region of $2800 \sim 3600 \mathrm{~m}$ as an important food crops, has the characteristics of both high yield and short maturity. Highland barley is the only grain that can mature normally in the alpine area with an altitude of more than $4200 \mathrm{~m}[1]$.

Artemisinin is an allelochemical in the common weed Artemisia annua. Artemisia annua is widely distributed in China, as well as in the temperate, frigid and subtropical regions of Europe and Asia. Artemisia annua may cause the transfer of artemisinin to the soil, thereby affecting soil organisms and aquatic environment [2,3].Artemisinin released into the environment during the growth process of Artemisia annua werill produce allelopathy, and inhibit the growth of surrounding plants to a certain extent. However, whether artemisinin has an allelopathic effect on highland barley is not well understood. 
Freeze-thaw refers to a physical geological effect and phenomenon in which the soil layer freezes and thaws when the temperature falling below zero and rising above zero. Freeze-thaw is the main ecological limiting factor in areas such as the Qinghai-Tibet Plateau. Long-term low temperature will cause tissue damage to plants, which in turn affects the growth and development of plants. It is revealed that the adaptability of highland barley seedlings to adversity environment is the key to cultivating highland barley varieties with strong cold resistance, which is helpful to take effective measures to reduce the harm caused by low temperature to plants.

The effects of freeze-thaw of plant are often accompanied by other ecological stresses, this study uses the freeze-thaw and artemisinin compound stresses that are widespread in the Qinghai-Tibet Plateau in spring as the influencing factors. Taking the highland barley seedlings as experimental materials, the physiological response characteristics under different concentrations of artemisinin stress, this study was conducted to explored the mechanism of physiological influence of both the single and multiple stress of freeze-thaw and artemisinin, in order to provide a theoretical basis for the scientific planting and management of highland barley.

\section{Materials And Methods}

\section{Materials}

The experiment was carried out in the laboratory of the School of New Energy and Environment of Jilin University in 2019. The highland barlery seeds were provided by the Life Science of Northeast Normal University of China. Barley seeds with full and uniform grain size were selected, disinfected with $0.1 \%$ $\mathrm{KMnO}_{4}$ for $2 \mathrm{~h}$, rinsed with deionized water, cultured in a tray containing wet filter paper, and $500 \mathrm{ml}$ Hoagland nutrient solution was added. It was cultured in $20^{\circ} \mathrm{C}$ light incubator (MGC-450BP) for $24 \mathrm{~h}$, then arranged neatly on a $40 \mathrm{~cm} \times 30 \mathrm{~cm}$ (long $\times$ wide) tray. The outdoor growth environment was simulated with the light incubator of which the germination condition was set as $12 \mathrm{~h}$ light $\left(25^{\circ} \mathrm{C}\right)$ and $12 \mathrm{~h}$ non-light $\left(15^{\circ} \mathrm{C}\right)$, and Hoagland nutrient solution was used to supply water once every day, each $40 \mathrm{ml}$. Cultivated for 7 days when the seedlings grow to $15 \mathrm{~cm}$, and selected seedlings with the same growing vigor for the experiment

\section{Methods}

\section{Preparation of artemisinin solution and Freeze-Thaw Treatment}

Weighed the dry crystalline artemisinin and dissolved it in the Hoagland nutrient solution, added a small amount of acetone to aid solubilization, stirred and added to the volumetric flask to prepare the artemisinin solution of $0 \mathrm{mg} / \mathrm{L}, 10 \mathrm{mg} / \mathrm{L}, 30 \mathrm{mg} / \mathrm{L}$ and $50 \mathrm{mg} / \mathrm{L}$, respectively. 
The samples of highland barley seedlings that require freeze-thaw stress in the experiment were marked and then subjected to freeze-thaw treatment. The other groups were still cultured in the incubator. The initial temperature of the test was $15^{\circ} \mathrm{C}$, The temperature dropped steadily to $-5^{\circ} \mathrm{C}$ at a speed of $5^{\circ} \mathrm{C}$ every $2 \mathrm{~h}$, and then the temperature rose from -5 to $10^{\circ} \mathrm{C}$ at same speed. The corresponding freeze-thaw sampling temperature was $10^{\circ} \mathrm{C},-5^{\circ} \mathrm{C}$, and $10^{\circ} \mathrm{C}$ (denoted as $\mathrm{T} 1-\mathrm{T} 3$, respectively) At each temperature, samples were taken from freeze-thaw treatments and non-freeze-thaw treatments at random according to the required amount of the measurement. The freeze-thaw machine was started at 6 am on the day of the experiment. After 2 hours, the first batch of samples were taken and randomly sampled. After that, samples were taken every 6 hours, and then taken twice, with three parallel samples each time. A total of 24 groups of samples in 3 time periods for index determination, divided into T1 (room temperature group at $8 \mathrm{am}$ ), T2(room temperature group at $2 \mathrm{PM}$ ), T3(room temperature group at $8 \mathrm{PM}), \mathrm{T} 1$ (D) $\left(10^{\circ} \mathrm{C}\right.$ freeze-thaw group at $8 \mathrm{am}), \mathrm{T} 2(\mathrm{D})\left(-5^{\circ} \mathrm{C}\right.$ freeze-thaw group at $\left.2 \mathrm{PM}\right), \mathrm{T} 3(\mathrm{D})\left(10^{\circ} \mathrm{C}\right.$ freeze-thaw group at 8 PM).

\section{Determination}

Soluble protein content in seedlings was determined by Coomassie Brilliant Blue method [4] after grinding the leaves into tissue homogenate and centrifuging. Malondialdehyde (MDA) content and soluble sugar content was measured with thiobarbituric acid chromatometry method (TBA) [5]. The SOD and CAT activity was measured with the enzyme kit produced by the Institute of Nanjing Jiancheng Biological Engineering. Using a photosynthesis measuring instrument to determine the net photosynthetic rate. Each indicator had 3 sets of parallel samples to calculate the average value

\section{Data Analysis}

The experimental data were graphed with Microsoft (Redmond, USA) Excel, and statistical analysis was performed with R 3.3.1 statistical software (R Foundation for Statistical Computing, Vienna, Austria). using single factor variance analysis (one-way analysis of variance) and multiple comparisons with least significant difference (LSD). The significance level was at 0.05 , the experiments were repeated five times, and all of the results are presented as mean \pm SE.

\section{Results And Analysis}

\subsection{Effects of Freeze-Thaw and Artemisinin Stress on MDA Content in Highland Barley Seedlings}

According to Fig. 1, the MDA content under freeze-thaw stress treatment was lower than that of the control group, which decreased by $9.0-40.9 \%$. Under the same other conditions, the freeze-thaw treatment led to the consumption of MDA in the barley leaves. The results were in good consistent with Fan Yuzhen's research on the response of white clover to low temperature stress [6]. Malondialdehyde (MDA) 
is one of the products of cell membrane lipid peroxidation, Its production can exacerbate membrane damage. Therefore, the increase of MDA concentration under normal temperature conditions is not conducive to the metabolism of highland barley seedling cells. The lower the temperature, the greater the damage to the membrane, and the stronger the membrane lipid peroxidation. Under the single freezethaw conditions and T3 sampling time, MDA content was the lowest, which was $4.9589 \mu \mathrm{mol} / \mathrm{g}$. MDA content decreased in both freeze-thaw and thaw-freeze stages, indicating that under freeze-thaw stress, MDA content in the body of highland barley seedlings gradually decreased in order to adapt to the environment and gradually repair the damage of cell membrane lipid.

Under the single artemisinin stress treatment, when the artemisinin concentration was $10 \mathrm{mg} / \mathrm{L}$, the MDA content was lower than without artemisinin treatment. As the artemisinin concentration increased, the MDA concentration also increased, indicating that the appropriate concentration of artemisinin is beneficial to the growth of highland barley. With the increase of the concentration of highland barley, the resistance of highland barley was weakened, so the content of MDA in the body increased, which was supported by the research results of Wei Liben et al.'s allelopathy on artemisinin on pasture [7].Under freeze-thaw treatment, when the artemisinin concentration was $0 \mathrm{mg} / \mathrm{L}$, there was little difference from the $10 \mathrm{mg} / \mathrm{L}$ MDA concentration. One important conclusion from our results was that freeze-thaw stress and artemisinin stress have antagonistic effects under certain conditions. The longer the treatment time, the higher the artemisinin concentration and the lower the MDA content in the seedlings. Therefore, the effect of artemisinin on highland barley seedlings is stronger than that of freeze-thaw.

\subsection{Effects of freeze-thaw and artemisinin stress on soluble sugar content in highland barley seedlings}

The increase in the amount of osmotic adjustment substances can alleviate or resist the damage caused by low temperature, and its content can reflect the strength of the plant's cold resistance ability [8]. As shown in Fig. 2, under a single freeze-thaw stress, the soluble sugar content of the freeze-thaw group was lower than that of the control group. In the freeze-thaw group at T1 (D), the soluble sugar content in the leaves was the highest, reaching a peak of $3.4349 \mu \mathrm{mol} / \mathrm{L}$. Kamata et al. found that plants increase the osmotic pressure by increasing the soluble sugar content and reduce the harm caused by low temperature stress [9]. After T2(D) and T3(D) continue to decrease, they decreased by $6.25 \%$ and $55.88 \%$ respectively $(P<0.05)$. It revealed that compared with the thaw-freeze stage, the freeze-thaw stage has a bigger effect on the soluble sugar content in the leaves of the seedlings. Compared with the control group, the soluble sugar content of the freeze-thaw group was reduced by $29.83 \%, 23.54 \%$, and $21.04 \%$ $(P<0.05)$. On the basis of our findings, it can be concluded that freeze-thaw hindered the transportation of soluble sugar and inhibited the accumulation of soluble sugar in the leaves of barley seedlings, resulting in a decline in the growth rate of seedlings and yellow and soft leaves. This was consistent with the observation from the results of Zhang Yue's research on the physiological regulation mechanism of white clover in temperature adaptation in different seasons [10].

After the artemisinin stress, the soluble sugar content showed different changing trends with the artemisinin concentration and action time. With the accumulation of the effect of artemisinin on the 
leaves, the soluble sugar content in the leaves was the lowest at T3 time. When the artemisinin concentration was between $0-10 \mathrm{mg} / \mathrm{L}$, the highland barley seedlings were the most sensitive and the soluble sugar content in the leaves decreased. Compared with the control group, when the artemisinin concentration was between $10-30 \mathrm{mg} / \mathrm{L}$, the highland barley seedlings appeared stress-resistant, and the soluble sugar content in the leaves increased. This was in accord with the research results of Lai Jieqing et al.on the effect of artemisinin on wheat seed germination and seedling growth [11]. Our results confirm that when the concentration was higher than $30 \mathrm{mg} / \mathrm{L}$ and less than $50 \mathrm{mg} / \mathrm{L}$, the leaf resistance was not enough to resist the influence of high concentration of artemisinin, and the leaves were damaged.

\subsection{Effects of freeze-thaw and artemisinin stress on soluble protein content in highland barley seedlings}

Figure 3 shows that compared with the control group, the soluble protein content of leaves in the freezethaw group increased first and then decreased. This was supported by the results of Zhou Ruilian on the physiological response of white clover and red clover to artificial stress [12]. In the thawing phase, the leaf cell membrane permeability increased and the content of osmotic regulators increased. During the freezethaw phase, cell membrane permeability decreased. As time goes on, the soluble protein in the leaf cells of highland barley seedlings remained at a relatively stable content, which coincided with with Fleck et al. on the stable protein accumulation and frost resistance of winter wheat leaves [13]. The results are further highlighted here that the barley seedlings have strong cold resistance, can produce protective substances when subjected to external stimuli, and are suitable for growth in cold environments.

When the concentration of artemisinin was at $10 \mathrm{mg} / \mathrm{L}$ level, the soluble protein content remained relatively stable with the change rate of only $2.4-6.0 \%$ over time(Fig. 4). At this time, the leaves of barley seedlings were maintained at a higher level of soluble protein, which was conducive to the growth of barley seedlings. This was inconsistent with the observation from the results of Wang Shuo et al. on the mechanism of allelopathy on wheat seedlings [14]. This difference may arise from that this experiment used different time periods to measure the soluble protein of highland barley seedlings, and the change of time and light conditions would affect the photosynthesis of the highland barley seedlings, resulting in the consumption of soluble protein.

Under the combined stress of freeze-thaw and artemisinin, the soluble protein content in the leaves of barley seedlings changed significantly. Compared with the control group, the soluble protein content of leaves under the compound stress treatment increased to different degrees. During the thaw-freeze process (T1-T2), the change of artemisinin concentration had little effect on the soluble protein content, while in the freeze-thaw phase (T2-T3), the soluble protein content first increased and then decreased(Fig. 5). When the concentration of artemisinin was $10 \mathrm{mg} / \mathrm{L}$, freezing and thawing stress had little effect on the soluble protein content of barley seedlings, from the results we had obtained, one can conclude that the barley seedlings have a certain degree of stress resistance and and the stress resistance was the strongest in artemisinin culture medium at $10 \%$ concentration.

\subsection{Effects of freeze-thaw and artemisinin stress on CAT activity in highland barley seedlings}


CAT is one of the main protective enzymes in plants. It has the function of scavenging active oxygen free radicals and can reduce the damage of active oxygen to plants [15]. CAT enzyme can promote the decomposition of $\mathrm{H} 2 \mathrm{O} 2$ produced in plants due to environmental changes to alleviate the damage caused by adversity stress [16]. Antioxidant enzyme system is an effective mechanism to resist low temperature stress [17]. As shown in Fig. 6, artemisinin and their combined stresses. Under single artemisinin stress, the CAT activity of the control group first increased from $12.2804 \mathrm{U} / \mathrm{mgprot}$ to $12.6422 \mathrm{U} / \mathrm{mgprot}$ (increased by $2.95 \%$ ) at $\mathrm{T} 1$ and then decreased to $7.2031 \mathrm{U} / \mathrm{mgprot}$ (decreased by 41.34\%) at T2 and T3. It showed that the concentration of artemisinin increased gradually, increasing by $13.78 \%$ and $21.79 \%$ respectively $(\mathrm{P}<0.05)$; after the adaptation of $\mathrm{T} 1 \sim \mathrm{T} 2$ time, the resistance ability of highland barley seedlings gradually increased, and CAT activity during T2 T3 gradually recovered. It must also be mentioned that under a single freeze-thaw stress, the CAT activity in the barley seedlings in

the freeze-thaw group continued to decrease during the thaw-freeze and freeze-thaw processes, indicating that the freeze-thaw process would lead to a decrease in CAT enzyme activity. Under the combined stress of freeze-thaw and artemisinin, the CAT activity in the barley seedlings were $33.28 \%$ lower than those in the control group. Under single artemisinin stress, each concentration group showed a decrease first and then an increase, and after freeze-thaw stress, then showed a gradual decrease. On the basis of these results we concluded that freeze-thaw will enhance the inhibitory effect of artemisinin on the growth of highland barley seedlings, making the compound stress more inhibitory to the growth of highland barley seedlings and lasting longer. While CAT activity appears at $50 \mathrm{mg} / \mathrm{L}$, the rebound growth reached 13.5598 $\mathrm{U} / \mathrm{mgprot}$, and the decrease in CAT activity decreased at $0-30 \mathrm{mg} / \mathrm{L}$. The behavior of the correlation coefficient makes us conclude that the resistance of highland barley seedlings was significantly enhanced, and an inflection point of inhibition may appear between $30-50 \mathrm{mg} / \mathrm{L}$.

\subsection{Effects of freeze-thaw and artemisinin stress on SOD activity in highland barley seedlings}

SOD is one of the important cell protection enzyme defense systems in plants [18],and it is the first line of defense to protect plant cells from reactive oxygen species. It can convert superoxide anion free radicals into $\mathrm{H}_{2} \mathrm{O}_{2}$ and protect plants from hydroxyl free radicals. Its activity directly reflects the ability of plants to be exposed to and tolerate reactive oxygen stress. The negative effects of various environmental stresses are at least partly due to the production of reactive oxygen species (ROS) and the suppression of the systems that protect them [19]. During the freeze-thaw treatment stage, the SOD activity of seedling leaves decreased first and then increased under both $0 \mathrm{mg} / \mathrm{L}$ and $30 \mathrm{mg} / \mathrm{L}$ artemisinin stress. At $10 \mathrm{mg} / \mathrm{L}$, it first increased and then decreased. When the artemisinin concentration was $50 \mathrm{mg} / \mathrm{L}$, it always showed a downward trend. When the artemisinin concentration was $0 \mathrm{mg} / \mathrm{L}$, compared with the CK group, the freeze-thaw group decreased by $16.90 \%$ at T2 and increased by $4.22 \%$ at T3; at $10 \mathrm{mg} / \mathrm{L}$, frozen Compared with the CK group, the thawing group increased by $29.51 \%$ at $\mathrm{T} 2$ and $11.44 \%$ at T3; at $30 \mathrm{mg} / \mathrm{L}$, compared with the CK group, T2 and T3 decreased by $25.03 \%$ and $12.55 \%$ respectively $(P<0.05) ; 50 \mathrm{mg} / \mathrm{L}$ compared with the CK group, the freeze-thaw group increased by $0.32 \%$ at $\mathrm{T} 2$ and decreased by $6.77 \%$ at T3(Fig. 7). It shows that the barley seedlings are more resistant to stress under freeze-thaw stress, and can maintain normal growth within a certain temperature range through their own metabolism. According 
to the research of Li Chen et al, the activity of SOD increased with decreasing temperature and reached a peak at $-2^{\circ} \mathrm{C}$. In this experiment, due to the large sampling temperature span, it can only be seen that the overall trend first decreased and then increased. It was impossible to determine the specific peak value. For each moment corresponding to T1, T2, and T3, the SOD activity of CK group changed with the change of artemisinin concentration. When the concentration increased from $0 \mathrm{mg} / \mathrm{L}$ to $10 \mathrm{mg} / \mathrm{L}$, the SOD activity decreased; from $10 \mathrm{mg} / \mathrm{L}$ when increased to $30 \mathrm{mg} / \mathrm{L}$, SOD activity increased and was greater than the activity at $0 \mathrm{mg} / \mathrm{L}$; when increased from $30 \mathrm{mg} / \mathrm{L}$ to $50 \mathrm{mg} / \mathrm{L}$, SOD activity decreased. From the results we have obtained, one can conclude that with the increase of artemisinin concentration, the stress resistance reflected by SOD of highland barley first decreases and then increases.

For different stages of the freeze-thaw group, as the concentration of artemisinin increased from $0 \mathrm{mg} / \mathrm{L}$ to $10 \mathrm{mg} / \mathrm{L}$, SOD activity decreased; when the concentration increased from $10 \mathrm{mg} / \mathrm{L}$ to $30 \mathrm{mg} / \mathrm{L}$, SOD activity increased; the concentration increased from $30 \mathrm{mg} / \mathrm{L}$ to $50 \mathrm{mg} / \mathrm{L}$, SOD activity decreased. On the surface, in the range of artemisinin concentration of about $0 \mathrm{mg} / \mathrm{L}-30 \mathrm{mg} / \mathrm{L}$, highland barley seedlings could maintain normal growth through metabolism. It is speculated that $50 \mathrm{mg} / \mathrm{L}$ artemisinin would cause greater damage to the seedlings and could not pass effectively. Metabolism removes harmful substances such as peroxides, so it cannot grow healthily. Zhu Hui-xia et al. pointed out that SOD, as an important component of the active oxygen scavenging system in plants, is closely related to the stress resistance of plants [20]. This is demonstrated in a number of studies that the change of SOD enzyme activity can be used as an evaluation index of plant cold tolerance. Mckerise B D et al. also proposed in the quantitative evaluation of chrysanthemum at the seedling and flowering stage that the SOD activity

of varieties with strong cold tolerance was higher, while the change trend of SOD activity of varieties with weak cold tolerance was not significant [21]. In general, freeze-thaw stress has little effect on SOD enzyme activity in highland barley seedlings, while artemisinin has a more obvious effect on SOD enzyme activity. The effects of both of them on SOD enzyme activity first increased and then decreased. The results were in bad consistent with Zhang Mengyu et al [22]. The results of Zhang Mengyu et al. first increased and then decreased. It should be pointed out that in this experiment, only the $30 \mathrm{mg} / \mathrm{L}$ concentration group without freeze-thaw satisfies this trend. The behavior of the correlation coefficient makes us conclude that the effect of artemisinin on highland barley Stress resistance has a great impact. Artemisinin can help highland barley alleviate freezing and thawing stress at low concentrations, while excessively high concentrations will increase the stress effect.

\subsection{Effects of freeze-thaw and artemisinin stress on net photosynthetic rate of highland barley seedlings}

Freeze-thaw stress can directly affect plant growth or indirectly affect plant growth by inhibiting photosynthesis. Freeze-thaw stress causes the intercellular icing to cause drought stress. Drought stress leads to a decrease in photosynthetic rate. There are stomata and non-stomata factors. The incomplete opening of stomata increases the resistance to $\mathrm{CO} 2$ diffusion to cells and decreases the photosynthetic rate; non-stomata factors Mainly cause serious damage to chloroplasts, decrease the vitality of the photosystem, and inhibit photosynthesis. The net photosynthetic rate reflects the accumulation of dry matter per unit time and unit area. 
It has been testified that freeze-thaw stress damages the photosynthetic function of plants [23]. To gain more insight qualitatively describe the effects of freeze-thaw artemisinin stress on the net photosynthetic rate of highland barley seedlings, the leaf $\mathrm{CO}_{2}$ metabolism rate $(\mathrm{A})$ and intercellular $\mathrm{CO}_{2}$ concentration (Ci) were selected as indicators of the net photosynthetic rate of highland barley seedlings. The ability of plants to use weak light at low temperatures is stronger than that of strong light, which leads to a reduction in the use of light energy by plant leaves, which provesd that temperature is the limiting factor for photosynthesis [24].

According to Fig. 8,under single artemisinin stress, as the concentration of artemisinin increases, leaf $\mathrm{CO}_{2}$ metabolism rate decreased first, then increased, and then decreased at T1, while at T2 and T3 it first increased, then decreased, and the overall decline trend. The intercellular $\mathrm{CO}_{2}$ concentration at $\mathrm{T} 1, \mathrm{~T} 2$, and T3 all showed a first decrease and then increase, and the increase in T1, T3 was greater than the decrease. This indicates that the presence of artemisinin prevents the stomata of highland barley seedlings from being fully opened, which increases the resistance to the diffusion of $\mathrm{CO}_{2}$ from the outside to the cells, and reduces the photosynthetic rate; non-stomatal factors mainly cause serious damage to chloroplasts, decreased photosystem activity, and photosynthesis. The effect was inhibited, the intercellular $\mathrm{CO} 2$ concentration increased, the photosynthetic capacity of the barley seedlings was weakened, and the net photosynthetic rate decreased. The results were in good consistent with Lai Qingjie [25], artemisinin promotes the growth of seedlings at low concentrations, while at higher concentrations it inhibits photosynthesis of plants. There are deviations due to different plants in the experiment, taking the above experimental results into account, when the concentration has an inflection point between $10 \mathrm{mg} / \mathrm{L}-30 \mathrm{mg} / \mathrm{L}$, the vicinity of the inflection point has an inhibitory effect, while other concentrations (less than $50 \mathrm{mg} / \mathrm{L}$ ) have a promoting effect.

Figure 9 shows that under a single freeze-thaw stress, the A value of the control group decreased sharply first, and then increased, while the A value of the freeze-thaw group was lower than that of the control group during the same period, and showed a downward trend, but the decline was small; The Ci value of the control group and the freeze-thaw group showed a trend of first increasing and then decreasing. Compared with the control group, the Ci value of the freeze-thaw group was $41.98 \%$ higher at $\mathrm{T} 1,22.57 \%$ lower at T2, and $43.25 \%$ lower at T3. It showed that the thaw-freeze process closed the stomata in the barley seedling leaves to a certain extent, the $\mathrm{Ci}$ value increased and photosynthesis weakened; while during the freeze-thaw process, the stomata in the barley seedling leaves gradually opened, the Ci value decreased, and photosynthesis increased. Under the combined stress of freeze-thaw and artemisinin, the A value of barley seedlings in the freeze-thaw group was almost all lower than that of the control group, while the $\mathrm{Ci}$ value was almost all higher than that of the control group, indicating that the combined stress would reduce the photosynthetic rate of barley seedlings. This was consistent with the observation from Yixin's results on the effects of freeze-thaw stress on rye seedlings[26].

\section{Conclusion}


The combined stress of freezing and thawing and artemisinin stress will cause the SOD and CAT enzyme activities in the barley seedlings to first decrease and then increase, the photosynthesis will be weakened overall, and the soluble sugar and soluble protein will first decrease and then increase, while the MDA content will increase overall under the circumstances, each group still has a trend of increasing first and then decreasing. The results of this study show that highland barley seedlings have strong cold tolerance. It must also be mentioned that artemisinin still has a strong allelopathic effect on highland barley seedlings under freezing and thawing conditions, and has a greater impact on its growth and development. Physiological effects are manifested as incomplete synergy, and highland barley seedlings showed strong resistance to stress. Given that this study is a short-term freeze-thaw stress, the physiological effects of different temperature gradients and freeze-thaw durations as well as different freeze-thaw intensities on highland barley seedlings under the condition of constant artemisinin concentration can be considered in the future experiments.

\section{Abbreviations}

MDA

malondialdehyde

CAT

catalase

SOD

superoxide dismutase

TBA

thiobarbituric acid chromatometry

LSD

least significant difference

ROS

reactive oxygen species

A

leaf $\mathrm{CO} 2$ metabolism rate

$\mathrm{Ci}$

intercellular $\mathrm{CO} 2$ concentration

\section{Declarations}

Ethics approval and consent to participate

Not applicable.

\section{Consent to publish}

Not applicable 
Availability of data and materials

All data generated or analyzed during this study are included in this

published article and its supplementary information files.

\section{Competing interests}

The authors declare that they have no conflict of interests.

\section{Funding}

This work was sponsored by the National Natural Science Foundation of China (Grant Nos. 31772669 and 32071874) and the 111 Project (B16020)

\section{Authors' Contributions}

$\mathrm{GB}$, and $\mathrm{KL}$ designed the experiments; $\mathrm{LK}, \mathrm{RF}, \mathrm{SZ}, \mathrm{XZ}$ and $\mathrm{XZ}$ performed most of the experiments and analyzed the data; and $Y C$ and $J Z$ wrote the manuscript. All authors agree with the manuscript contents and with its submission. All authors read and approved the final manuscript.

\section{Acknowledgements}

Not applicable.

\section{References}

1. Li XP. Root rot diseases of highland barley in Qinghai-Tibet Plateau and their effects on rhizosphere soil microecology [D].Gansu Agricultural University,2017.

2. Jessing KK, Cedergreen N, Jensen J, Hansen, Hans CB. DEGRADATION AND ECOTOXICITY OF THE BIOMEDICAL DRUG ARTEMISININ IN SOIL[J]. Environmental Toxicology and Chemistry,2009,28(4).

3. Zhi-Qiang Yan ,Dan-Dan Wang,Lan Ding,Hai-Yan Cui,Hui Jin,Xiao-Yan Yang,Jian-She Yang,Bo Qin. Mechanism of artemisinin phytotoxicity action: Induction of reactive oxygen species and cell death in lettuce seedlings[J]. Plant Physiology and Biochemistry,2015,88.

4. Chen J, Tao L, Zhu W. Biochemical experiment. 3rd ed. Beijing: Science; 2004. pp. 13-4.

5. Wang X. Principle and technology of plant physiological and biochemical experiments. 2nd ed. Beijing: Higher Education; 2010. pp. 190-281. (in Chinese).

6. Fan YZ. Response of Trifolium trifolium to Low Temperature Stress [J].Jiangsu agricultural science, 2009, (1), 231-232233. The doi: 10.3969 / j.i SSN. 1002-1302.2009.01.095.

7. Wei LB, Xia ZL, Yin J, Yuan L. Allelopathic effects of artemisinin on herbage [J]. Acta Prataculturae Sinica. 2017;26(05):62-9. 
8. Koehler G, Wilson RC, Goodpaster JV,et a1.Proteomic study of low-temperature responses in strawberry cultivars (Fragariaxananassa that differ in cold tolerance[J].P1ant Physiol,2012,159(4): 1787-1805.

9. Kamata T, Uemura M. Solute accumulation in heat seedlings during cold acclimation: contribution to increased freezing toler囚ance[J]. Cryo Letters. 2004;25(5):311-22.

10. Zhang Y. Response of white clover at different leaf ages to temperature in different seasons and its physiological plasticity [D].Ludong University,2016.

11. Lai QJ, Zhao WY, Li XL, et al. Effects of artemisinin on seed germination and seedling growth of wheat [J].Journal of Triticeae Crops,2016,36(10):1386-1390.

12. Zhou Ruilian Z, Mei Z, Ping, et al.Physiological differences between white clover and red clover in response to artificial thaw stress [J]. Chinese Journal of Ecology, 2012,(6).1334-1340.

13. Fleck RA, Day JG,Clarke $\mathrm{K} J$,et a1.Elucidation of the metabolic and structural basis for the cryopreservation recalci- trance of Vaucheria sessilis[J].Cryo-Letters,1990,20: 271-282.

14. Wang S, Mu X, Yang C, et al.Journal of Northwest A\&F University (Social Science Edition),200634(6):106-110.

15. Bowley SR, Mc Kerise. B D.Relationships among freezing,low temperature flooding,and ice encasement tolerance in al-falfa[J].Can.J.Plant Sci.1990,70: 227-235.

16. Li Chen B, Guozhang CC, Revolution L, Ziyong. Li Yanfang.Physiological responses of ryegrass seedlings to freeze-thaw and acid deposition [J]. Jiangsu Agricultural Sciences,2017,45(08):159162.

17. Li J, Yan XF, Zu YG. Generation of Activated Oxygen and Change of Cell Defense Enzyme Activity in Leaves of Korean Pine Seedling Under Low Temperature[J]. Acta Botanica Sinica. 2000;42(2):14852.

18. Chen KT, Renaut J, Sergeant K, et al Proteomic Changes Associated with Freeze-Thaw Injury and Post-Thaw Recovery in Onion(A llium cep a L.)Scales[J].Plant,Cell \& Environment,2013,36(4):892905.

19. BOR M, ÖZDEMIR F, TüRKAN I. The effect of salt stress on lipid peroxidation and antioxidants in leaves of sugar beet Beta vulgaris L and wild beet Beta maritime L.[J]. Plant Sci. 2003;164(1):77-84.

20. Zhu HX, Sun WC, Deng B, et al. Study on Cold Hardiness and Its Physiological and Biochemical Characteristics of Winter Turnip Rape(Brassica campetris)[J]. Acta Agriculturae Boreali-Occidentalis Sinica, 2007:436-438.

21. Mckerise BD, Murnaghan J, Jones KS. Iron-superoxide dismutase expression in transgenic alfalfa increases winter tolerance[J].PlantPhysiol,2000,122:1427-1437.

22. Zhang MY. Physiological effects of alkaline salt stress and mowing disturbance on rye under freezethaw environment [D].Jilin University,2020.

23. Steffen KL, Arora R, Palta JP. Relative sensitivity of phoyosynthesis and respiration to freeze-thaw stress in herbaceous species: Importance of realistic freeze-thaw protocols [J]. Plant Physiol. 
1989;89(4):1372-9.

24. Sarhan F, Ouellet F, Vazquez-Tello A. The wheat WCS90 gene family: a useful model to understand the molecular genetics of freezing tolerance in cereals[J]. Physiol Plant. 1997;101(2):439-45.

25. Lai QJ. Effect of artemisinin on seed germination and seedling growth of wheat and barley [D].Liaoning Normal University,2017.

26. Chang YX. Physiological response characteristics of rye seedlings to drought and freeze-thaw stress [D]. Jilin University,2020.

\section{Figures}

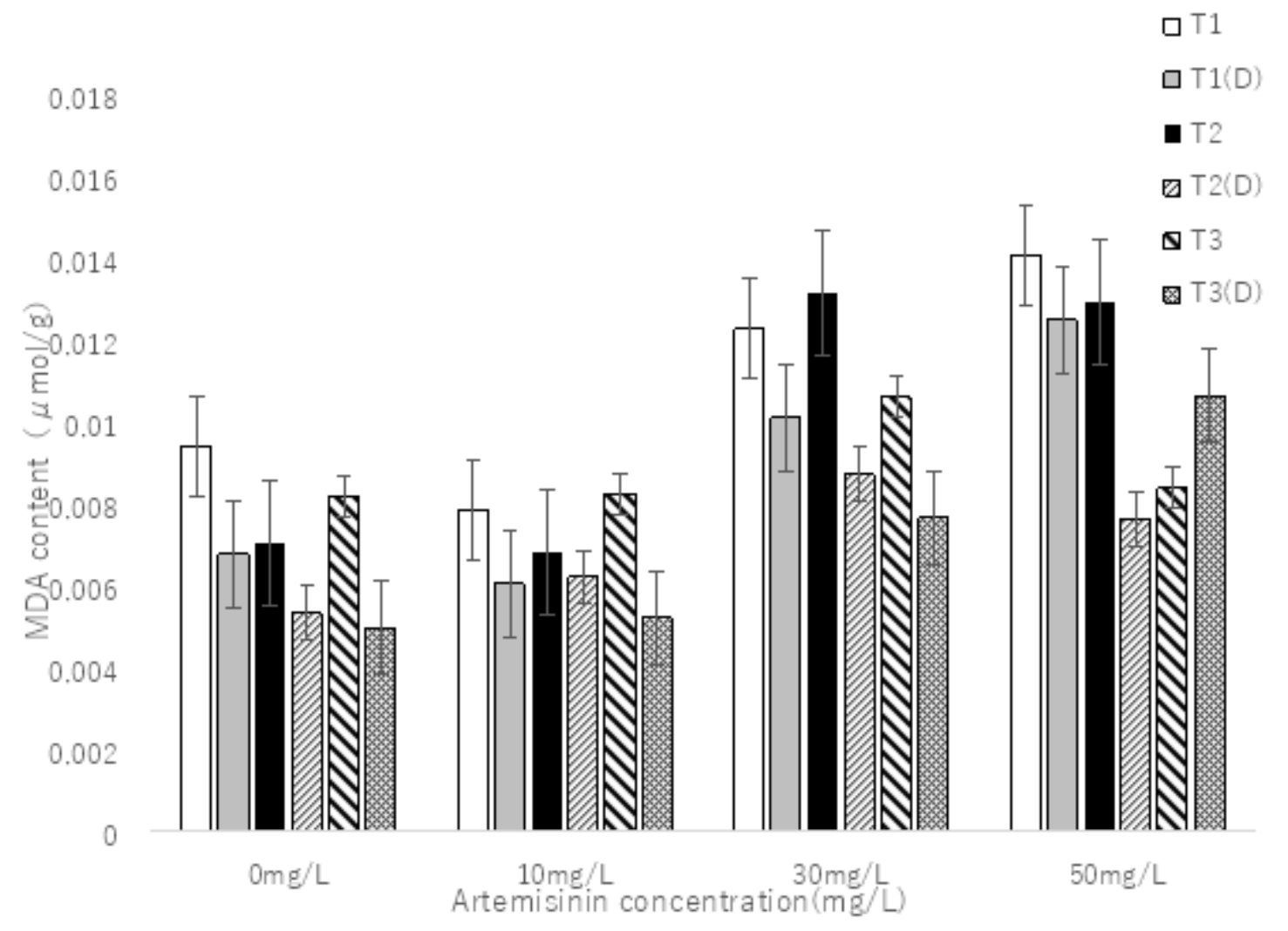

Figure 1

Effects of different artemisinin concentrations on MDA content of highland barley seedlings under freeze-thaw stress. T1(D)-T3(D) represent 2 nd, 8 th and 14 th respectively, corresponding to temperatures $10,-5$,and $10^{\circ} \mathrm{C} ; \mathrm{T} 1-\mathrm{T} 3$ represent $2 \mathrm{nd}, 8$ th and 14 th respectively, corresponding to room temperatures. 


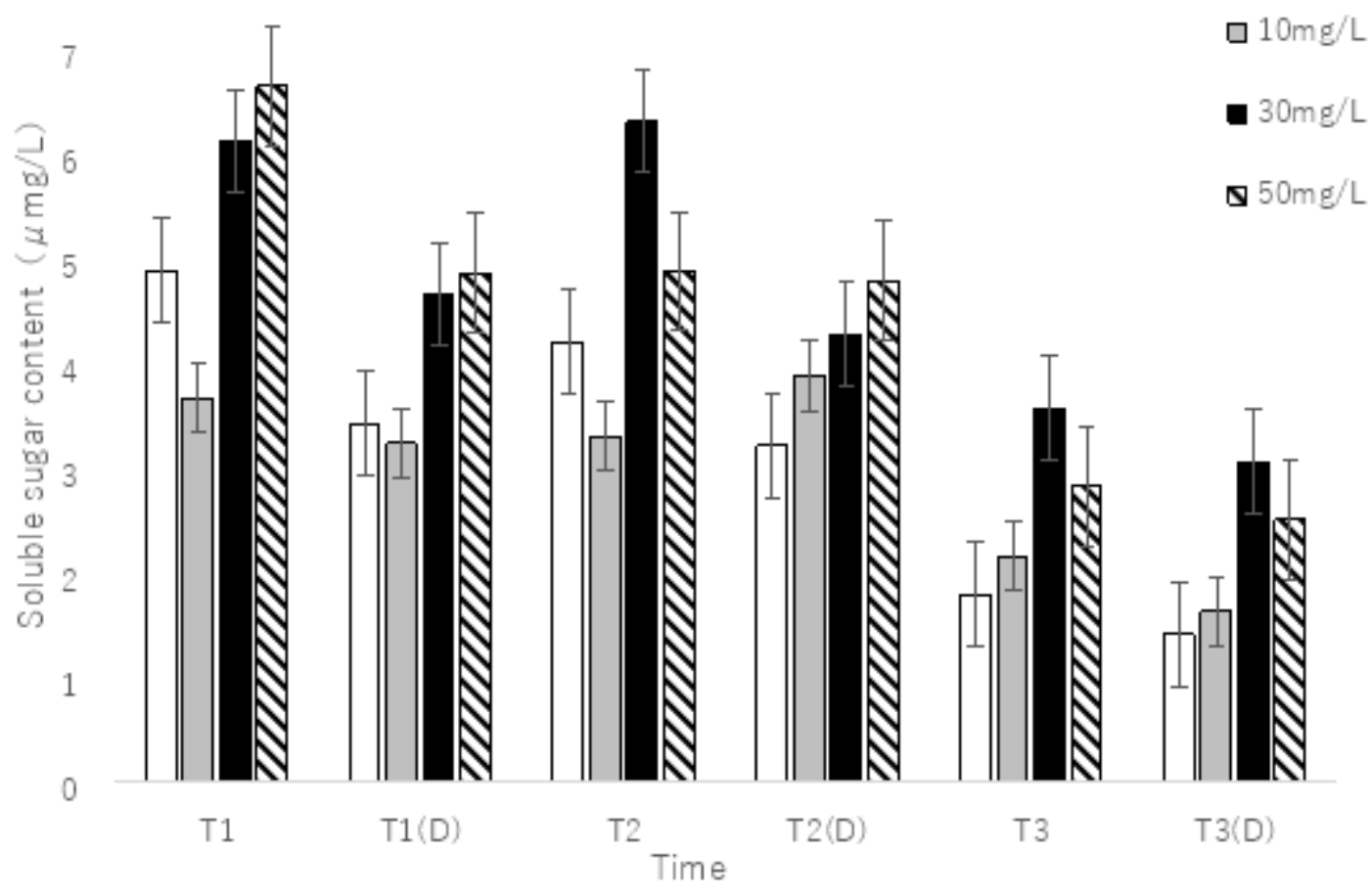

Figure 2

Effects of different artemisinin concentrations on soluble sugar content of highland barley seedlings under freeze-thaw stress. T1(D)-T3(D) represent 2nd, 8th and 14th respectively, corresponding to temperatures $10,-5$, and $10^{\circ} \mathrm{C}$; $\mathrm{T} 1-\mathrm{T} 3$ represent 2 nd, 8 th and 14 th respectively, corresponding to room temperatures;0,10,30,50 represent artemisinin concentration.

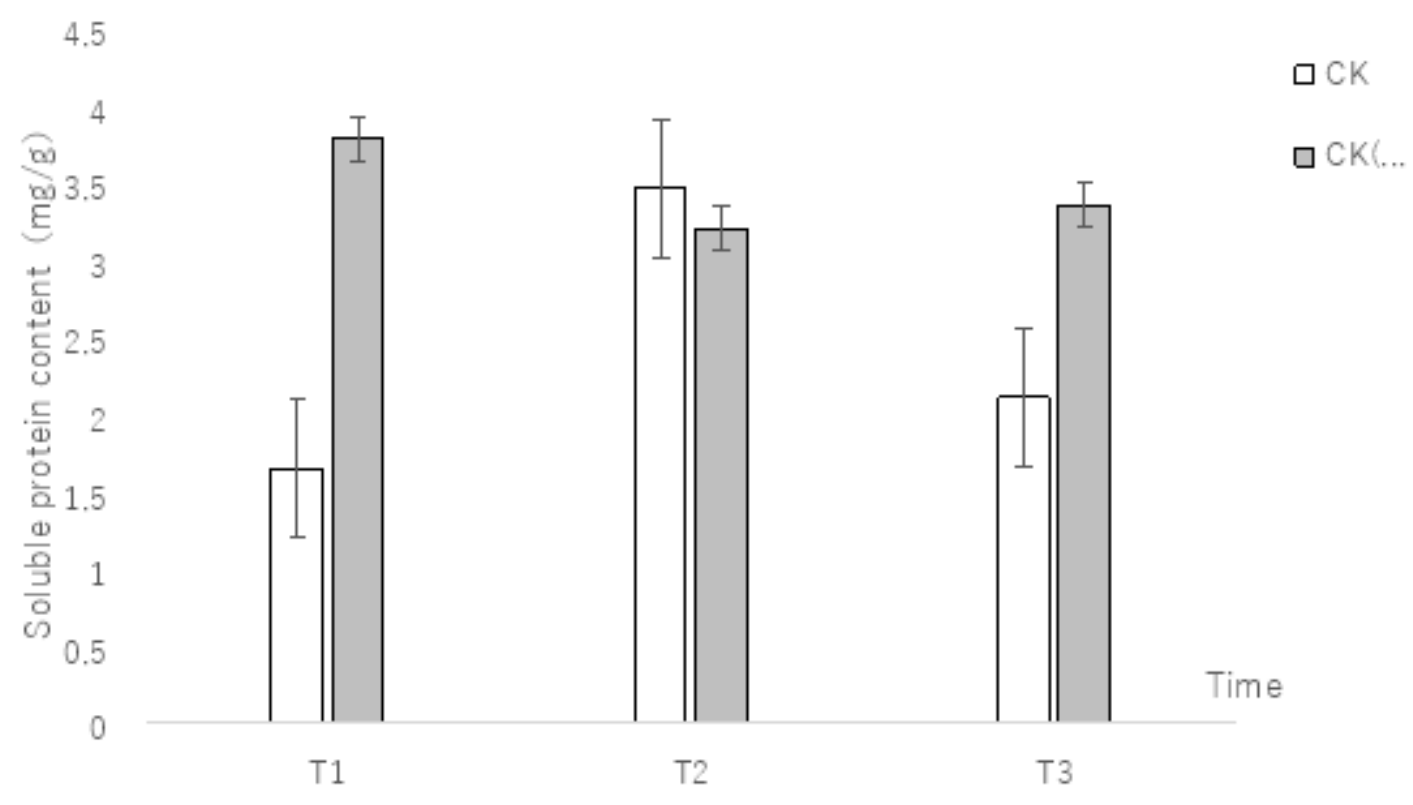

Figure 3 
Effects of freeze-thaw stress on soluble protein content of highland barley seedlings. T1-T3 represent 2 nd, 8 th and 14 th respectively, corresponding to temperatures $10,-5$,and $10^{\circ} \mathrm{C}$; CK is control group.

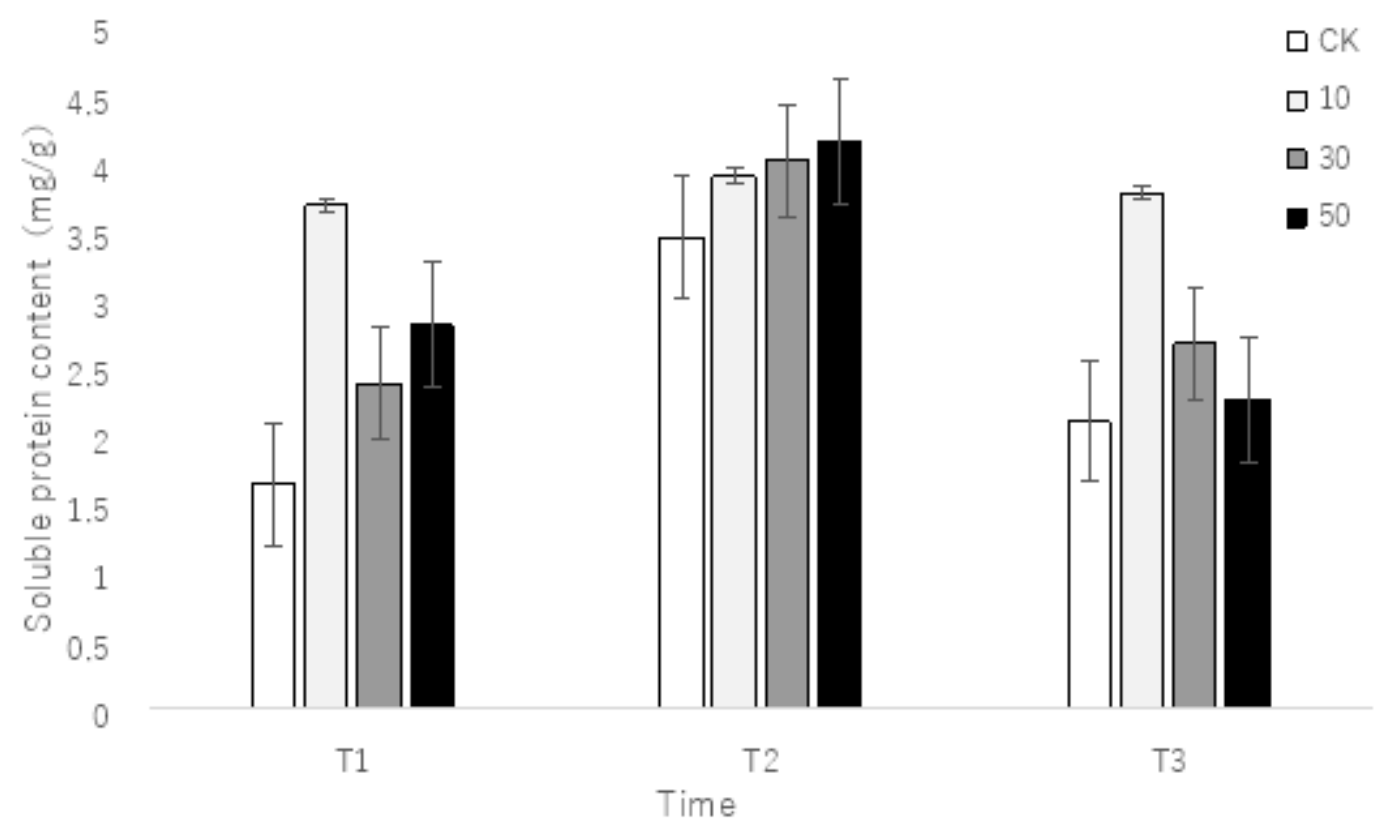

Figure 4

Effects of different artemisinin concentrations stress on soluble protein content of highland barley seedlings. T1-T3 represent 2nd, 8th and 14th respectively, corresponding to temperatures $10,-5$, and $10^{\circ} \mathrm{C}$; CK is control group; $10,30,50$ represent artemisinin concentration.

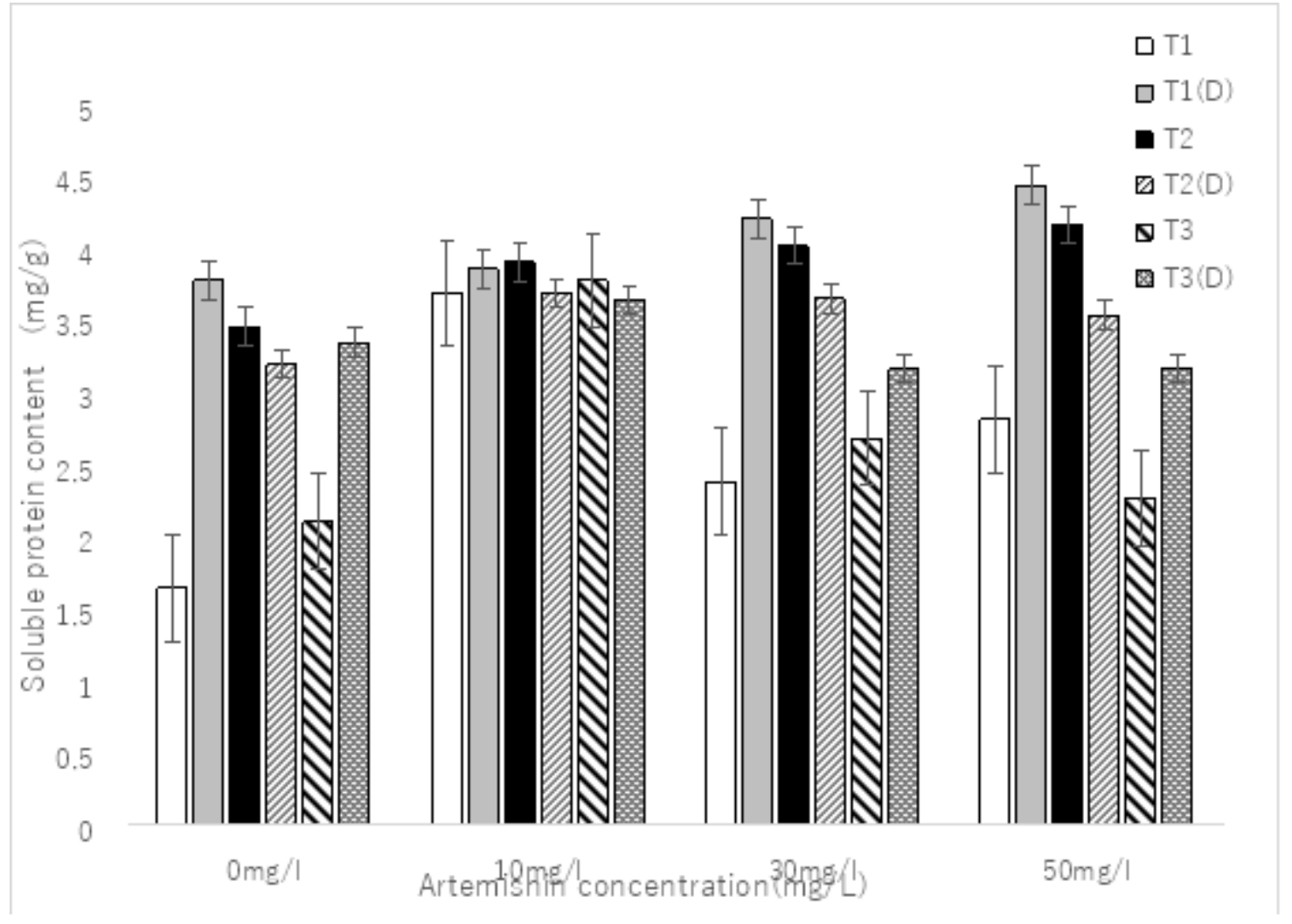

Figure 5 
Effects of different artemisinin concentrations on soluble protein content of highland barley seedlings under freeze-thaw stress. T1(D)-T3(D) represent 2nd, 8th and 14th respectively, corresponding to temperatures $10,-5$, and $10^{\circ} \mathrm{C} ; \mathrm{T} 1-\mathrm{T} 3$ represent $2 \mathrm{nd}$, 8 th and 14 th respectively, corresponding to room temperatures.

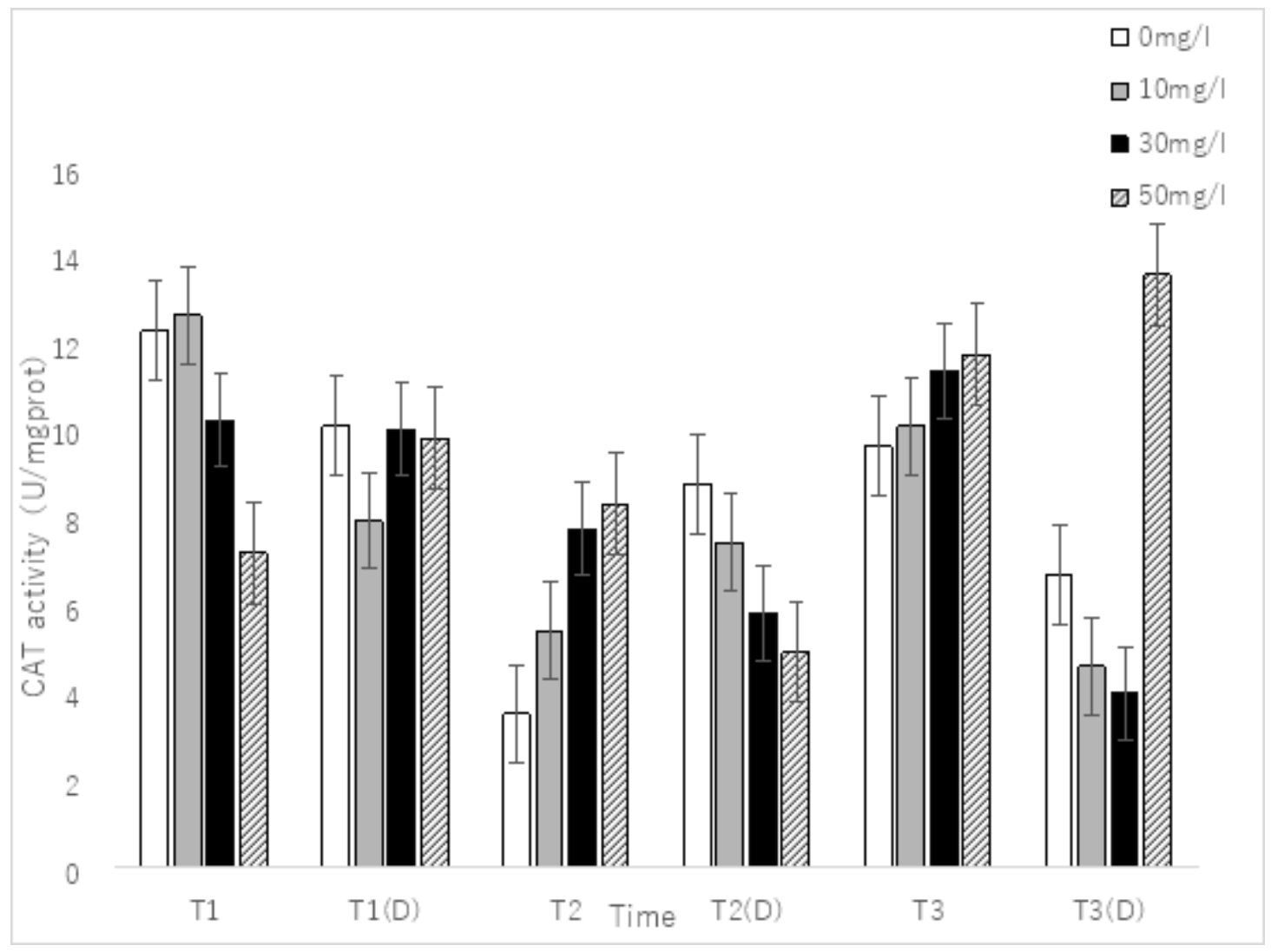

Figure 6

Effects of different artemisinin concentrations on CAT activity of highland barley seedlings under freezethaw stress. T1(D) $-T 3(D)$ represent 2 nd, 8 th and 14 th respectively, corresponding to temperatures $10,-5$,and $10^{\circ} \mathrm{C}$; T1 $-\mathrm{T} 3$ represent $2 \mathrm{nd}$, 8 th and 14 th respectively, corresponding to room temperatures. $0,10,30,50$ represent artemisinin concentration. 


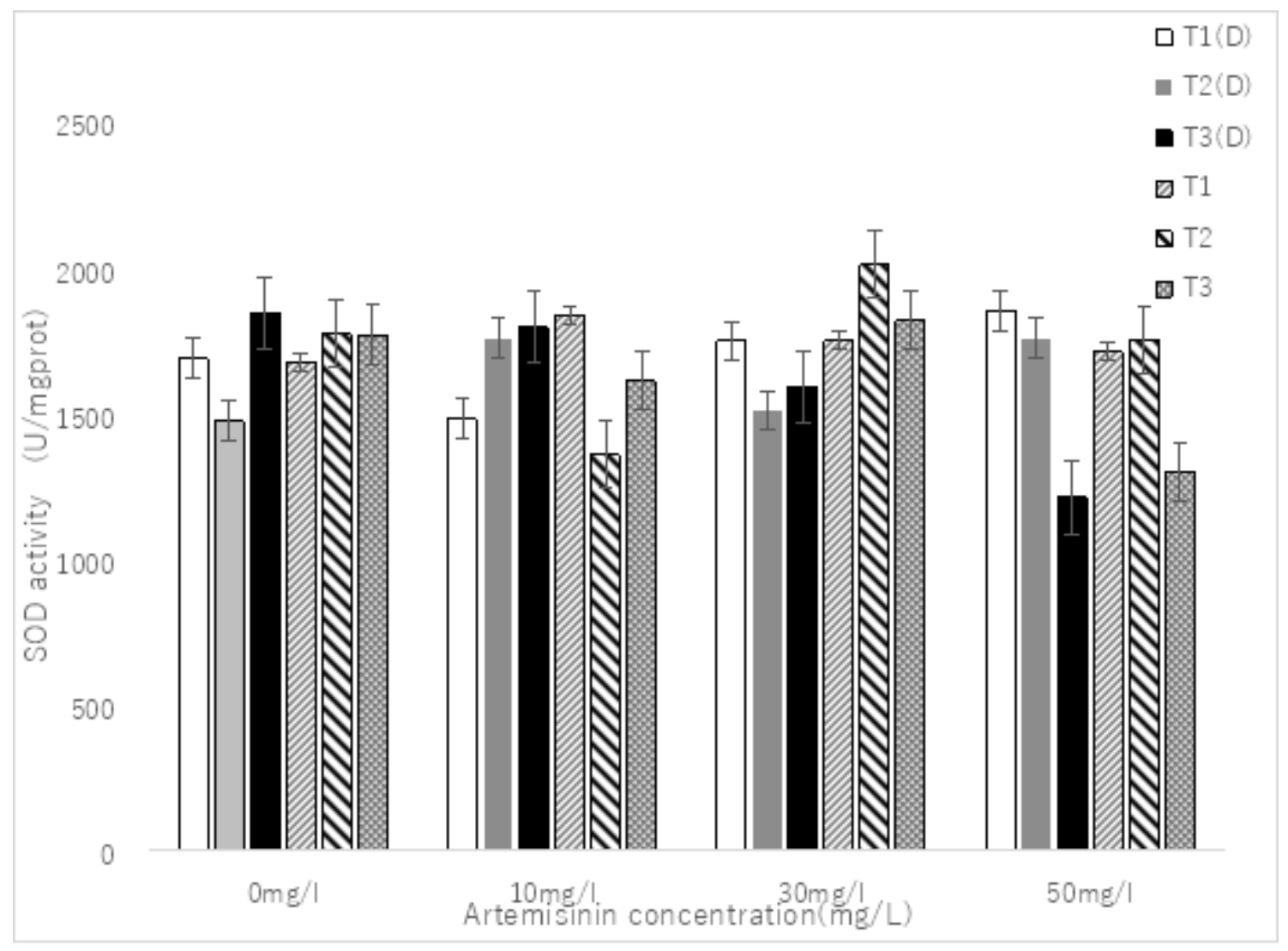

\section{Figure 7}

Effects of different artemisinin concentrations on SOD activity of highland barley seedlings under freeze-thaw stress T1(D)-T3(D) represent the 2 nd , 8 th and 14 th, $C$; corresponding to temperature $10,-5$ and $10 \otimes \mathrm{T} 1-\mathrm{T} 3$ represent $2 \mathrm{nd}$, 8 th and 14 th respectively, corresponding to room temperature. $0,10,30,50$ represents artemisinin concentration.

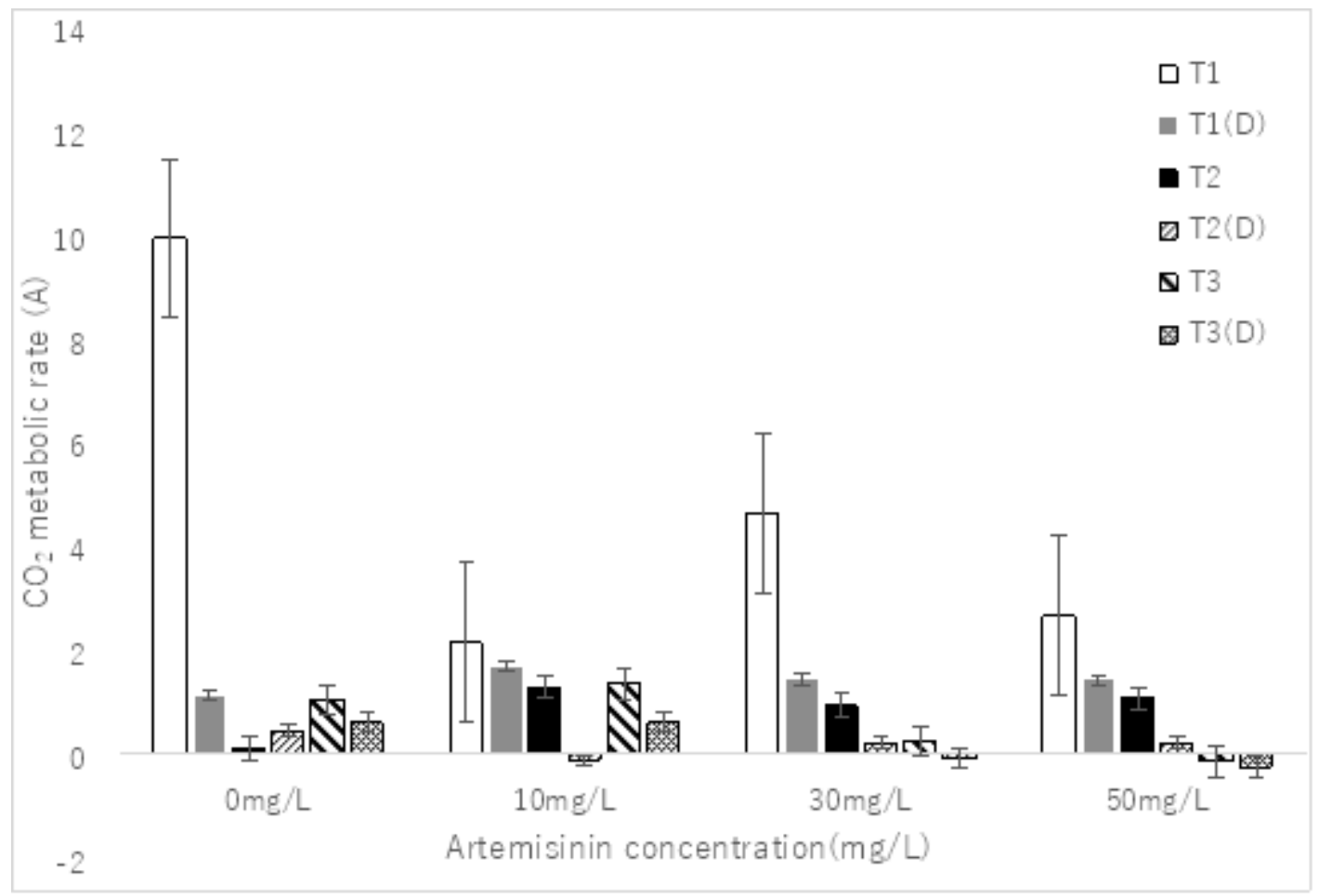




\section{Figure 8}

Effects of different artemisinin concentrations on $\mathrm{CO} 2$ metabolic rate of highland barley seedlings under freeze-thaw stress. T1(D)-T3(D) represent the 2 nd, 8 th and 14 th respectively, C; corresponding to temperature 10,-5 and 10冈T1-T3 represent 2nd, 8th and 14th respectively, corresponding to room temperature. $0,10,30,50$ represents artemisinin concentrations.

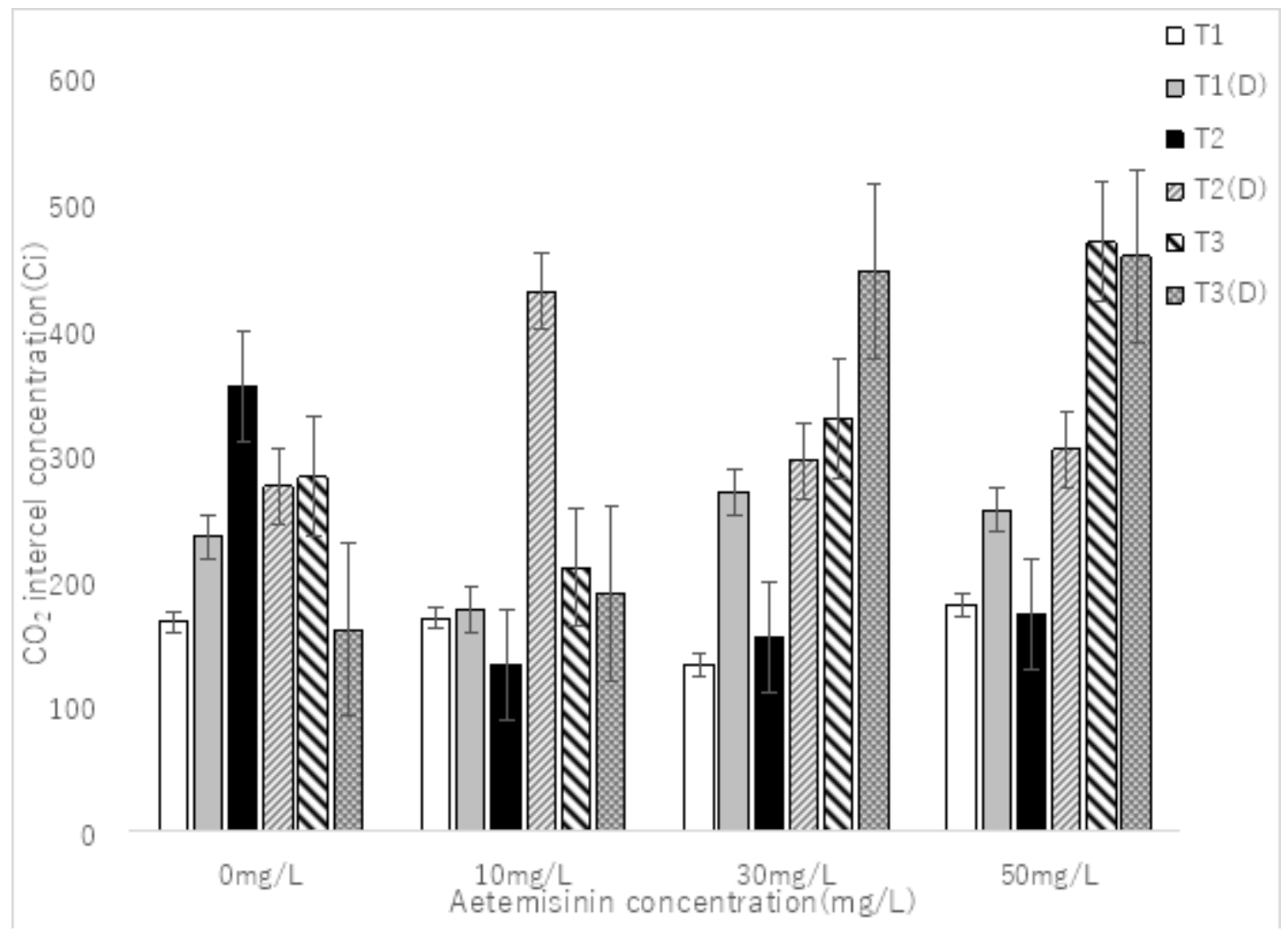

Figure 9

Effects of different artemisinin concentrations on intercellular CO2 concentration of highland barley seedlings under freeze-thaw stress.T1(D)-T3(D) represent the 2nd, 8th and 14th respectively, C; corresponding to temperature $10,-5$ and $10 \otimes \mathrm{T} 1-\mathrm{T} 3$ represent 2 nd, 8th and 14th respectively, corresponding to room temperature. $0,10,30,50$ represents artemisinin concentrations. 